\title{
Congenital Unicuspid Aortic Valve Repair without Cusp Patch Augmentation
}

\author{
Jama Jahanyar ${ }^{1}$, Gaby Aphram ${ }^{1}$, Daniel E. Munoz ${ }^{1}$, Stefano Mastrobuoni ${ }^{1}$, Emiliano \\ Navarra $^{1}$, Laurent de Kerchove ${ }^{1}$, and Gebrine Elkhoury ${ }^{1}$ \\ ${ }^{1}$ Cliniques universitaires Saint-Luc
}

February 22, 2022

\begin{abstract}
Unicuspid aortic valves are rare congenital malformations. Surgical repair is feasible in aortic regurgitation, and in some cases of aortic stenosis. The standard surgical approach is a bicuspidization and symmetrization with pericardial patch augmentation of valve cusps. Herein, we are describing our original technique for bicuspidization of a unicuspid aortic valve without cusp patch augmentation. We also address the surgical management of a commissural diastasis.
\end{abstract}

\section{Introduction}

The aortic valve (AV) is a semilunar valve and is derived from endocardial cushions of the primitive heart tube. The right and left cusps stem from conotruncal cushions, whereas the non-coronary cusp originates from right-posterior intercalated cushions. Cavitation of the cushions leads to separation of the cusps with formation of a lumen, and this is followed by elongation and thinning of the cusps. Dysregulation of extracellular matrix remodeling for instance, can lead to valve malformations, and thus different valve phenotypes 1 .

The normal AV-phenotype is tricuspid, but we know that AVs can range from unicuspid (UAV) to quadricuspid valves, with even further variability beyond this spectrum ${ }^{2}$. Nonetheless, UAVs are rare congenital heart valve malformations, with an estimated prevalence of $0.02 \%{ }^{3}$. Although there is no official classification for UAVs, possibly due to its' rarity, we generally distinguish between an acommissural- and unicommissural phenotype(Figure1A,B) .

UAVs can be repaired through biscuspidization. This is generally achieved through patch augmentation of both cusps (e.g. butterfly patch), with or without realignment of the commissural angle or aortic annuloplasty 4, 5 . As in other AV-phenotypes, there is a spectrum of UAV morphologies. However, the commissure is usually positioned posteriorly (Figure1B) and the height of the two raphae is generally lower (5-15mm) than the level of the commissure ${ }^{6}$, as in bicuspid $\mathrm{AVs}_{\mathrm{s}}{ }^{6,7}$. Nonetheless, there is no one technique fits all approach for UAV-repair, and herein we are presenting our original technique for bicuspidization of a regurgitant UAV without the usual patch augmentation of the cusps.

\section{Technique}

The surgical technique is illustrated in the Video (Video 1, supplementary material). A transverse aortotomy is performed $1.5 \mathrm{~cm}$ above the origin of the right coronary artery, after the heart is arrested with normothermic blood cardioplegia. The valve is assessed and confirmed to be unicuspid with a true posterior commissure at the left/non-coronary commissural position, and two raphae at the non/right-, and right/left-position (Figure1C,D) . The height of both raphae is below the height of the true posterior commissure. An additional unusual finding is a diastasis of the posterior commissure, which had to be addressed separately (Figure2C). 
Although the aortic root is only moderately enlarged at $4.2 \mathrm{~cm}$, we performed our modified reimplantation technique, since it provides the best stabilization of the functional aortic annulus, with an excellent aortic annuloplasty and support of the Sino-tubular junction ${ }^{7}$.

The aortic root is prepared and annular sutures are placed at the level of the virtual basal ring as previously described by our group ${ }^{7}$. A commissurotomy is then performed at the non/right-raphe to create a second functional commissure, and the free margins are thinned with a blade (Figure2A,B). The remaining raphe is then shaven and thinned towards the hinge point of the anterior cusp, to allow for better mobility (Figure2C) . Attention has to be paid towards not accidentally perforating the cusp at its' insertion.

The commissural diastasis of the posterior commissure is then addressed. The commissure is incised towards its' tip, in order to readjust the width and to exclude some abnormal tissues in-between the cusps. The commissure is then remodeled with a 4.0 Prolene suture(Figure2D). This resolved the commissural diastasis and excluded the abnormal tissues in-between the cusps and led to improved apposition of cusp free margins.

A 30mm Valsalva-graft is then seated onto the aortic annulus and tied down. The commissure and modified raphe are resuspended at $180^{\circ}$. The nadir of the fused cusp, at the raphe, is lowered to match the nadir of the non-fused cusp (Video1). The graft is then sewn to the proximal aortic root remnant and the aortic valve is re-assessed thereafter. The anterior cusp is prolapsed and the neo-commissure is lower than the posterior commissure. We usually perform central cusp plications to treat a prolapse, but due to the good quality of cusp tissues in proximity to the neo-commissure, and the bulk of tissues centrally, we opted to perform a lateral cusp plication instead. This lateral plication at the neo-commissure can also help to somewhat elevate the commissure, if at the same time a small bite of the aortic wall is taken laterally (Figure $3 A, B$ ). In addition to this, the neo-commissure was further resuspended, $5-10 \mathrm{~mm}$ higher to match the height of the posterior commissure, utilizing a 5.0 Prolene suture with emphasis on the anterior prolapsing cusp (Figure $3 C, D$ ). This is feasible, due the good quality and large amount of cusp tissues, as well as the annuloplasty, which increases cusp mobility due to a relative increase of free margin length.

There was good coaptation, with an adequate effective- and geometric height thereafter. Both coronary buttons were then reimplanted in the usual fashion, and the distal aortic anastomosis was performed, resecting the diseased aorta and wrapping the very distal ascending aorta with a piece of prosthetic graft.

\section{Comments}

Unicuspid AVs are rare congenital malformations. However, the true prevalence of this valve morphology is likely underreported. For instance, Noly et al. reported that only $14 \%$ of patients with UAV were diagnosed preoperatively ${ }^{8}$. Even intraoperatively, UAV may remain under-recognized due to severe calcifications for instance; or inexperience of the surgeon or echocardiographer with identification of complex AV-morphologies.

Unicuspid AVs, without severe calcifications, can be repaired through bicuspidization. The standard technique is a butterfly patch repair ${ }^{4}$, with symmetrization of the cusps and improvement of cusp mobility, through additional modifications of the functional aortic annulus (e.g. annuloplasty, sinus plication), and thinning of the raphe.

In selected patients, Matsushima et al. have achieved a UAV-repair without patch material, through cusp nadir relocation and root remodeling. This achieved a relative elevation of the newly created commissure, which then more closely matched the height of the true commissure ${ }^{6}$. The reason for this approach is to obviate the accelerated degeneration of autologous or xenogenic pericardial patch material, which renders this a palliative repair approach, and thus only allows for deferment of valve replacement later in life. Nonetheless, this is still an advantage in children and adolescents, and we think it is also of benefit when employed in adults, as the better survival of valve repair compared to prosthetic valve replacement is now well documented. Moreover, this may also function as a bridge to a Ross-procedure later in life, in children but also in younger to middle-aged adults.

In this patient however, we performed an AV-repair without a patch, through commissurotomy of a raphe to create a second commissure and two symmetric cusps. We further enhanced symmetry through reimplan- 
tation of the commissures at $180^{\circ}$. Cusp mobility was improved through annuloplasty, as well as thinning of the free margins and raphe, and repair of a commissural diastasis. The anterior commissure was elevated through cusp plication and additional resuspension. Since no additional patch material was used, we hope that we were able to minimize the risk of cusp degeneration with overall improvement in valve longevity, similar to our bicuspid AV-repairs ${ }^{9}$.

\section{References}

1. Martin PS, Kloesel B, Norris RA, Lindsay M, Milan D, Body SC. Embryonic Development of the Bicuspid Aortic Valve. J Cardiovasc Dev Dis. 2015;2:248-272.

2. Jahanyar J, Aphram G, de Kerchove L, Khoury GE. A Unique Hybrid Tricuspid Aortic Valve. Ann Thorac Surg. 2021.

3. Novaro GM, Mishra M, Griffin BP. Incidence and echocardiographic features of congenital unicuspid aortic valve in an adult population. J Heart Valve Dis. 2003;12:674-678.

4. Lorenz V, Gonthier S, Jahanyar J, de Kerchove L, El Khoury G. Original repair after Ross failure: A case report of bicuspidized unicuspid autograft. JTCVS Tech. 2021;8:141-143.

5. Aicher D, Bewarder M, Kindermann M, Abdul-Khalique H, Schafers HJ. Aortic valve function after bicuspidization of the unicuspid aortic valve. Ann Thorac Surg. 2013;95:1545-1550.

6. Matsushima S, Hess A, Glenske M, Hoffmann K, Raddatz A, Schafers HJ. Cusp Nadir Relocation by Root Remodeling in Unicuspid Aortic Valve Repair. Ann Thorac Surg. 2019;108:e409-e412.

7. Jahanyar J, El Khoury G, de Kerchove L. Commissural geometry and cusp fusion insights to guide bicuspid aortic valve repair.JTCVS Tech. 2021;7:83-92.

8. Noly PE, Basmadjian L, Bouhout I, Viet Le VH, Poirier N, El-Hamamsy I. New Insights Into Unicuspid Aortic Valve Disease in Adults: Not Just a Subtype of Bicuspid Aortic Valves. Can J Cardiol. 2016;32:110116.

9. de Meester C, Vanovershelde JL, Jahanyar J, et al. Long-term durability of bicuspid aortic valve repair: a comparison of 2 annuloplasty techniques. Eur J Cardiothorac Surg. 2021.

\section{Figures}

Figure1. A, B. 2 Unicuspid valve morphologies; A. Acommissural Unicuspid Aortic Valve; B. Unicommissural Unicuspid Aortic Valve.C. Intraoperative assessment. D. Echo assessment (short axis).

Figure 2. A. Commissurotomy. B. Cusp shaving.C. Raphe thinning. D. Remodeling Commissural Diastasis.

Figure 3. A. Non-fused cusp lateral plication. B.Fused cusp lateral plication. C. Fused cusp elevation.D. Further commissural elevation.

\section{Supplementary material}

Video 1. Unicuspid aortic valve repair without patch material. 

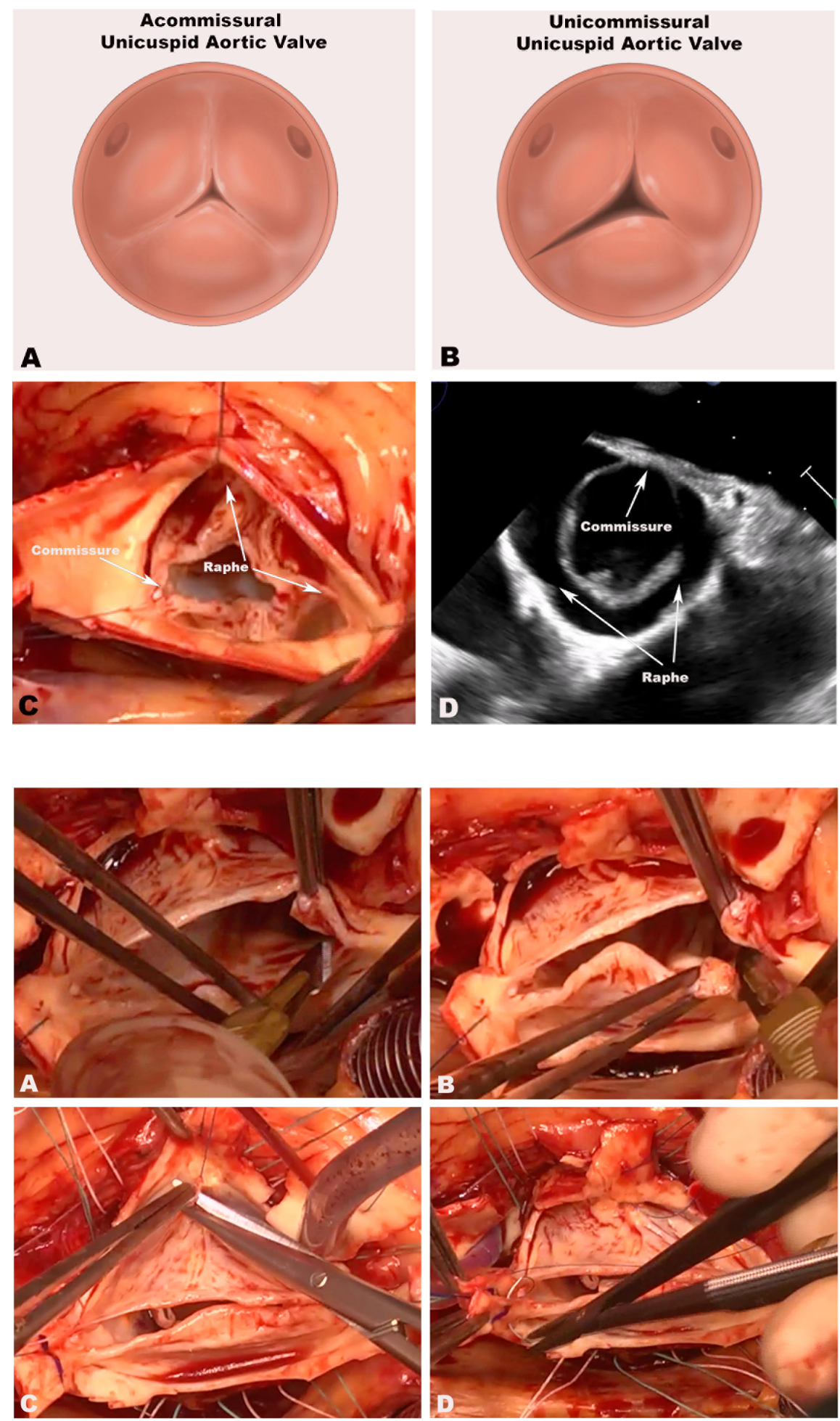

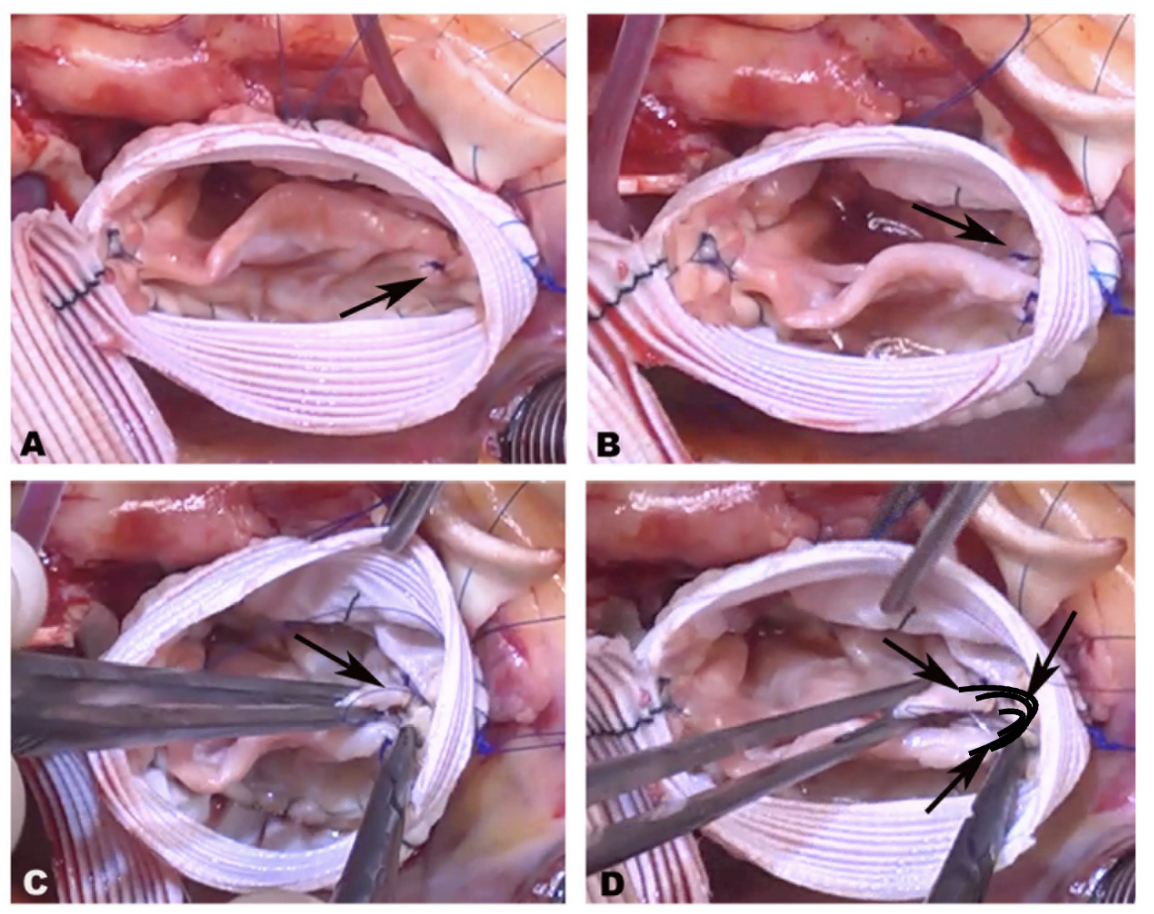